\title{
A Failure Analysis of Air Heat Exchanger Based on Modal Calculation and Thermal-Structural Interaction Simulation
}

\author{
Q. Miao, Z. Wang, and L. Wang
}

\begin{abstract}
This paper deals with the failure analysis of air heat exchanger. The natural frequency and vibration mode of air heat exchanger are obtained based on a three-dimensional finite element model. A flow-induced vibration is analyzed on the basis of modal simulation results. A thermal-structural interaction simulation for air heat exchanger is carried out by using the multi-physical field coupling method. The stress distribution and strain distribution of air heat exchanger are calculated by using the results of temperature field simulation. A Manson-Coffin model based on equivalent plastic strain range and cycle life is used to describe fatigue life, and the equivalent plastic strain range was obtained by the thermal-structural interaction simulation. The analysis results show that the major reason of fatigue failure of air heat exchange tube bundle is the thermal stress and flow-induced vibration.
\end{abstract}

Index Terms-Heat exchanger, failure, simulation, modal.

\section{INTRODUCTION}

Heat exchanger, one of the core components of heat exchange, is widely used in various industrial fields. In the working process, heat exchanger often breaks down due to high temperature, high pressure and other complex loading. In the actual project, there are many cases of failure of heat exchanger which caused loss and damage. The heat transfer efficiency is being improved by increasing the span and decreasing the rigidity of the tube bundle in recent years, at the same time the fluid flow rate is more and more fast, therefore, the failure of the tube bundles become the focus of attention. Gardner [1] had researched theoretically the effect of pressure load and thermal load to the heat exchanger tube bundle. Singh, Holtz [2], Ohol [3], Kasahara and Iwata [4] obtained the temperature field thermal stress distribution of heat exchanger by using a simplified finite element model of heat exchanger. Tube shell heat exchanger is the most widely used heat exchanger in engineering. El, Amjad [5], and J. X. Wu, Q. W. Dong, M. S. Liu [6] investigated the most serious failure of shell and tube heat exchanger were, heat exchange

Manuscript received March 12, 2016; revised June 7, 2016. This work was supported by "The Fundamental Research Funds for the Central Universities" No. DUT15QY35. A failure analysis of air heat exchanger based on modal calculation and thermal-structural interaction simulation.

The authors are with Key Laboratory of Ocean Energy Utilization and Energy Conservation of Ministry of Education, Dalian University of Technology, Dalian, 116024 China (e-mail: miaoqing@mail.dlut.edu.cn, zhengw@dlut.edu.cn, wanglu@dlut.edu.cn). tube bundle and tube plate connection failure, flow induced vibration of heat exchanger tube bundles, and heat exchanger tube bundle fracture. C. L. Liu and J. F. Luan [7] pointed out that the vibration, corrosion and material were heat exchanger main failure influence factors.

Fluid induced vibration of tube bundles has been becoming more and more obvious in heat exchanger failure. Fluid induced vibration of heat exchange components will lead to severe noise and damage of the components, which is obviously detrimental to the life of the heat exchanger Connors [8] presented a model for calculating the fluid induced vibration of the heat exchange tube in a steam generator. Katinas and Markevicius [9] believed that the transient fluid exciting force and the natural frequency of heat exchange tube directly affected the vibration of the heat exchange tube. However, some papers had also reported that thermal stress was one of the main causes of failure of tube bundles. Z. C. Wang, B. L. He [10] simulated flow and heat transfer process of high temperature heat exchanger under 5 kinds of working conditions by ANSYS, and evaluated strength with thermal fluid-structural interaction simulation results. C. Z. Guo and H. Zhou [11] obtained temperature distribution of heat exchange tube, shell and tube sheet by simulation, used the temperature data of each node as thermal load to the structure analysis, and calculated the thermal stress caused by temperature difference.

This paper deals with the failure analysis of air heat exchanger. The prediction method for the fatigue failure of the heat exchanger is provided to reduce the loss caused by the unexpected failure in the actual project. Based on a three-dimensional finite element model, the natural frequency and vibration mode of air heat exchanger were obtained, which was used in fluid induced vibration analysis. Also based on the finite element model, the heat exchanger temperature field distribution was simulated. The stress distribution and strain distribution of air heat exchanger were calculated by using the data of temperature field simulation. Through the series of work, the main failure reasons of heat exchanger were founded. At the same time, quantitative calculation for the fatigue life of the tube was obtained on basis of the stress field simulation results.

\section{NUMERICAL METHOD}

\section{A. Modal Calculation}

Vibration of the heat exchangercaused by the working process is simplified as a non-damped vibration. The basic equation of the typical nondamped modal analysis is as (1). 
This is a typical eigenvalue problem, the intrinsic properties of the structure are only determined by the stiffness matrix and mass matrix:

$$
[K]\left\{\varphi_{i}\right\}=\omega_{i}^{2}[M]\left\{\varphi_{i}\right\}
$$

where $[K]$ is stiffness matrix, $\left\{\varphi_{i}\right\}$ is modal vectors of the $i$ order modes, $\omega_{i}$ is the natural frequency of the i order modes, $[M]$ is mass matrix.

There are many methods can be used to solve the above equations. In this paper, the block Lanczos method of ANSYS software was used to solve. Lanczos algorithm is to achieve Lanczos recursive calculation with a set of vectors. This method is accurate and fast.

\section{B. Calculation of Fluid Induced Vibration Frequency}

In the shell side of heat exchanger, when the fluid flows transversely through the bundle, the main causes of flow induced vibration is: Carmen vortex, turbulence jitter and fluid elastic instability [12]. Carmen vortex frequency is determined according to (2):

$$
f_{v}=S_{t} V / d_{0}
$$

where $S_{t}$ is Strouhal number, $d_{0}$ is diameter of heat exchange tube, $V$ is cross flow velocity.

The main frequency of the turbulence is determined according to (3) [13]:

$$
f_{t}=V d_{0} K\left(1-d_{0} / t\right)^{2} / l t
$$

where $t$ is center distance of horizontal heat exchange tube, $l$ is center distance of longitudinal heat exchange tube, $K$ is constants.

The critical cross flow velocity is calculated by (4):

$$
V_{c}=K_{c} f_{n} d_{0} \delta_{s}{ }^{b}
$$

where $K_{c}$ is ratio coefficient, $f_{n}$ is natural frequency, $\delta_{s}$ is mass damping parameter.

According to the heat exchanger design manual, when the ratio of the minimum natural frequency of heat exchanger $f_{n}$ to the Carmen vortex frequency $f_{v}$ is greater than 0.5 , or the ratio of the minimum natural frequency of heat exchanger $f_{n}$ to the main frequency of the turbulence $f_{t}$ is greater than 0.5 , or flow velocity $V$ is greater than the critical cross flow velocity $V_{c}$, there is a possibility of tube bundle vibration.

\section{Predicting Method of the Fatigue Life}

The operating characteristic of the air heat exchanger was low cycle thermal fatigue, adopting strain amplitude to characterize. The famous fatigue life formula, Manson-Coffin formula, as (5) can be used to predict fatigue life:

$$
\Delta \varepsilon_{p} * N_{f}^{a}=C
$$

where $\Delta \varepsilon_{p}$ is plastic strain range, $N_{f}$ is fatigue life of specimen, $a$ and $C$ is material constants.

Formula (5) is rearranged as (6):

$$
\log N_{f}=\log C / a-\log \Delta \varepsilon_{p} / a
$$

From (6), plastic strain amplitude is linearly related to fatigue life. This evaluation criteriais widely used in engineering.

\section{NUMERICAL SIMULATIONS}

\section{A. Computational model}

The geometric size of the air heat exchanger is $200 \mathrm{~mm} \times 200 \mathrm{~mm} \times 375 \mathrm{~mm}$, which has 2534 heat exchange tubes, 4 pull rods, 2 tube sheets and 3 baffle plates. The diameter of heat exchanger tube is $2 \mathrm{~mm}$, while the length is $239 \mathrm{~mm}$. The material of tube bundle, pull rods, tube sheets and baffle plates are $1 \mathrm{Cr} 18 \mathrm{Ni}$ 9Ti.Three-dimensional finite element model was performed in commercial software Altair Hypermesh. Shell element was selected, and the number of elements generated was about 600 thousand as in Fig.1. The elements of tubes are shown in Fig. 2.

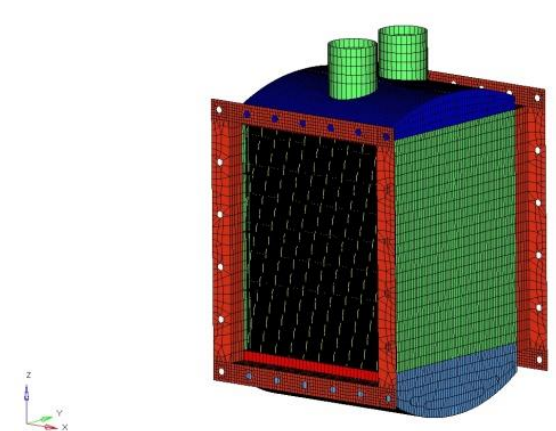

Fig. 1. Three dimensional finite element model of heat exchanger.

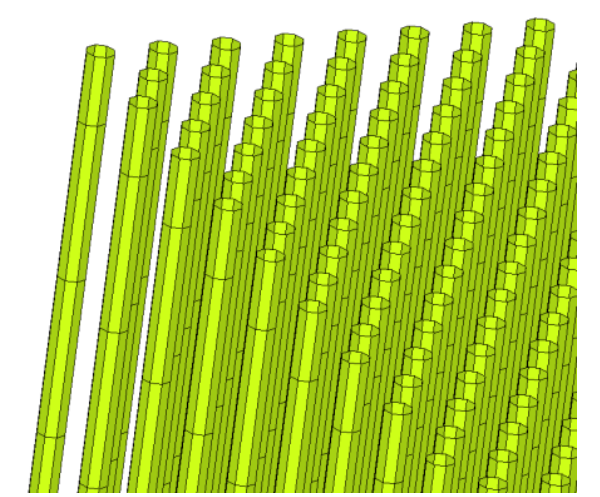

Fig. 2. Finite element model of heat exchanger tube bundles.

\section{B. Simulation Procedure}

Numerical simulation was completed by commercial software ANSYS.

In heat exchanger modal simulation, shell 63 element was selected. Because the structure was composed of thin shell elements, block Lanczos method was applied, at the same time, the lumped mass matrix is adopted to get faster and better results.

Temperature field analysis was obtained by using Steady-State Thermal module, loading the third kinds of temperature boundary conditions, with shell57 thermal analysis element. According to the results, temperature load was translated to the structure.

Stress field and strain field were obtained by Static Structural module, with shell131 element. Character of material properties varied according to the temperature was considered, nonlinear analysis was adopt.

\section{Boundary Conditions}

For the air heat exchanger in this paper, the working medium of the tube and shell are both air. Related process parameters are as in Table I. 
In heat exchanger modal simulation, fixed constraint was applied at the flange bolt holes. On the top and bottom tube sheets, tube and tube sheet share common nodes at the tube sheet contact position, the radial degrees of freedom of the heat exchange tube were constrained at the baffle plates contact position.

\section{TABLE I: HEAT EXCHANGER WORKING MEDIA}

\begin{tabular}{lcc}
\hline \hline & Tube side & Shell side \\
\hline Media & Air & Air \\
Working temperature $\left({ }^{\circ} \mathrm{C}\right)$ & $380_{-40} / 120_{-23}^{+5}$ & 85 \\
Maximum flow $(\mathrm{kg} / \mathrm{h})$ & $1450 \pm 50$ & $4000 \pm 100$ \\
\hline \hline
\end{tabular}

TABLE II: THE FIRST TWENTYORDER FREQUENCYOF HEAT EXCHANGER

\begin{tabular}{c|c|c|c}
\hline \hline Frequency $(\mathrm{Hz})$ & Order & Frequency $(\mathrm{Hz})$ & Order \\
\hline 432.98 & 1 & 452.79 & 2 \\
514.84 & 3 & 526.20 & 4 \\
554.29 & 5 & 576.78 & 6 \\
581.24 & 7 & 659.73 & 8 \\
711.27 & 9 & 719.96 & 10 \\
901.69 & 11 & 903.77 & 12 \\
925.53 & 13 & 936.65 & 14 \\
962.09 & 15 & 1014.9 & 16 \\
1017.2 & 17 & 1028.1 & 18 \\
1029.2 & 19 & 1031.9 & 20 \\
\hline \hline
\end{tabular}

In heat exchanger temperature field simulation, tube side was regarded as tube slot turbulent forced convection heat transfer, and shell side was fluid horizontal flow tube bundle of external forced convection heat transfer. Convective heat transfer coefficients were applied to the surface of tube sheets, tube bundle and shell respectively.

In heat exchanger static structural simulation, similarly fixed constraint was applied at the flange bolt holes. On the top and bottom tube sheets, tube and tube sheet share common nodes at the tube sheet contact position, the radial degrees of freedom of the heat exchange tube were constrained at the baffle plates contact position. The temperature load is imported and applied from the result of temperature field analysis.

\section{RESUlTS AND DISCUSSION}

\section{A. Model Analysis}

In the finite element analysis of the natural frequency of the heat exchanger, the first twenty order frequency were extracted, as shown in Table II. As a representative, the first order mode of the tubes is shown in the Fig. 3.

As a result, the Carmen vortex frequency of the air heat exchanger shell side flow field is:

$$
f_{v}=S_{t} V / d_{0}=32883.1 \mathrm{~Hz}
$$

The main frequency of the turbulent is:

$$
\mathrm{f}_{\mathrm{t}}=V d_{0} K\left(1-d_{0} / t\right)^{2} / l t=29140.29 \mathrm{~Hz}
$$

The critical cross flow velocity is:

$$
V_{c}=K_{c} f_{n} d_{0} \delta_{s}^{b}=234.33 \mathrm{~m} / \mathrm{s}
$$

As,

$$
f_{v} / f_{n}=75.94>0.5, f_{t} / f_{n}=67.30>0.5, V<V_{c}
$$

Based on the calculation results, the tube bundle vibration would be generated by the Carmen vortex or turbulent flow. The vibration would cause the collision between the tube bundle and the baffle plate, with a higher stress generated between the pipe wall and the baffle plate, which caused the failure of the heat exchange tube in the baffle plate because of abrasion or shearing.

As can be seen from Fig. 3, the vibration would start from the center of the tube bundle, because of the further distance from the fixed position constraint.

Of course, when the tube buddle is subjected to the excitation force of the flow field, the vibration mode, the starting position and so on will change. Therefore, it is needed to analyze the structural response and stress caused by fluid induced vibration, the next step, the transient response of the structure under the action of the flow field can help us to solve the related problems.

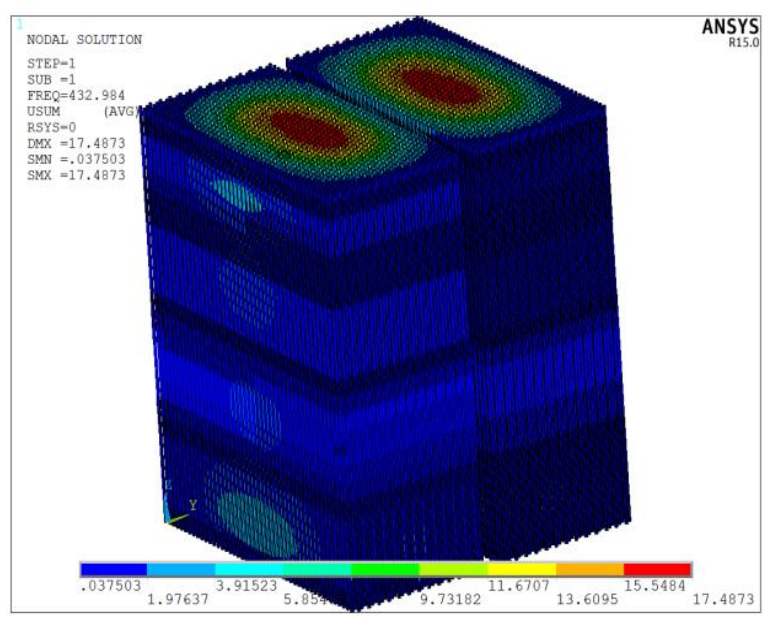

Fig. 3. The first order mode of the tubes.

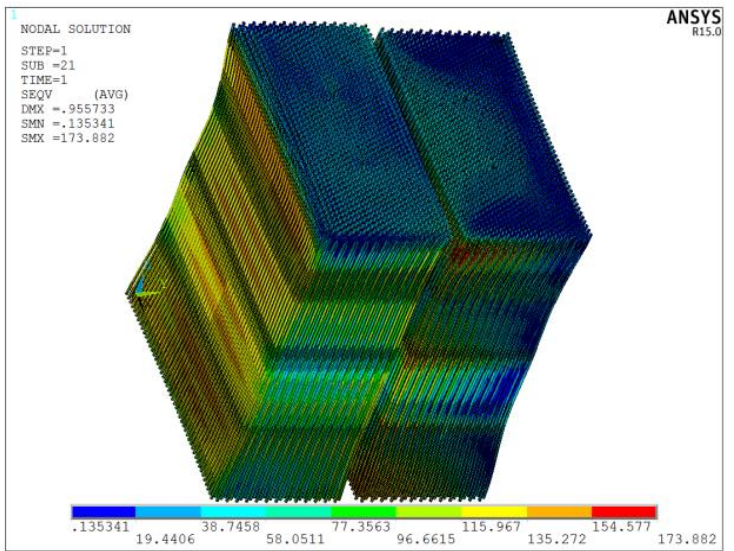

Fig. 4(a). Stress field distribution of the tubes.

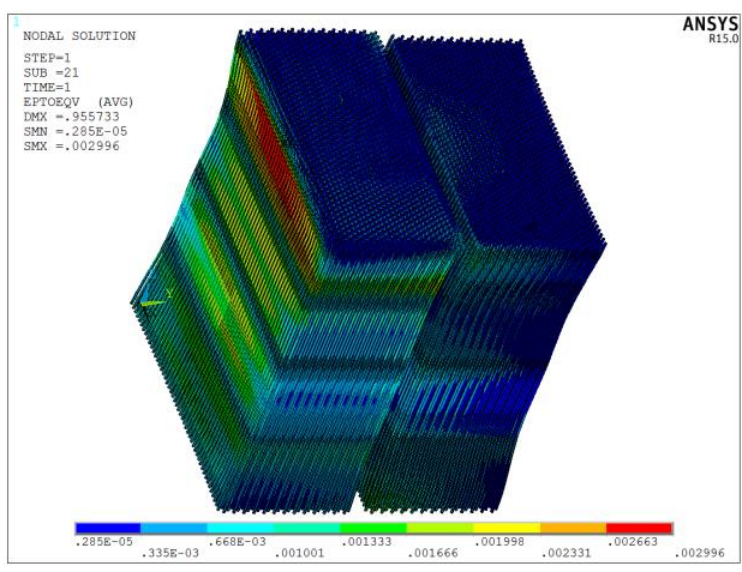

Fig. 4(b). Strain field distribution of the tubes. 


\section{B. Stress and Strain Field Analysis}

Under the temperature load, the stress field distribution of the tubes are shown in the Fig. 4(a), the strain field distribution and the plastic strain field distribution of the tubes are shown in the Fig. 4(b) and Fig. 4(c), and the stress field and the strain field distribution of the plates are shown in the Fig. 5(a) and Fig. 5(b).

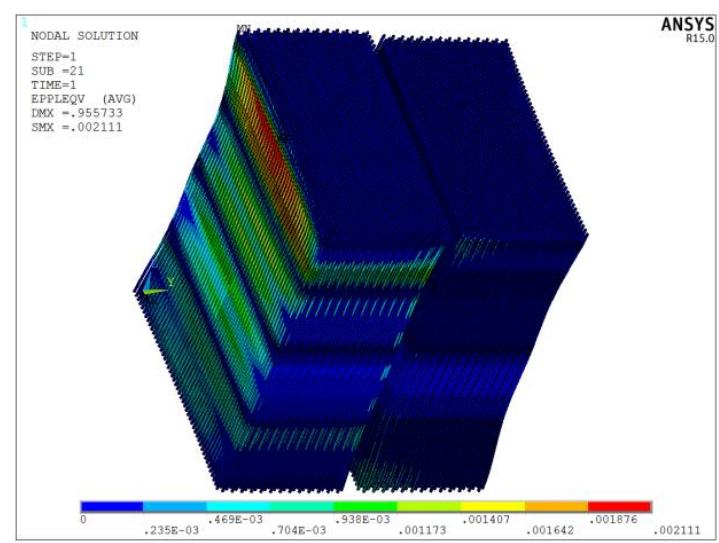

Fig. 4(c). Plastic strain field distribution of the tubes.

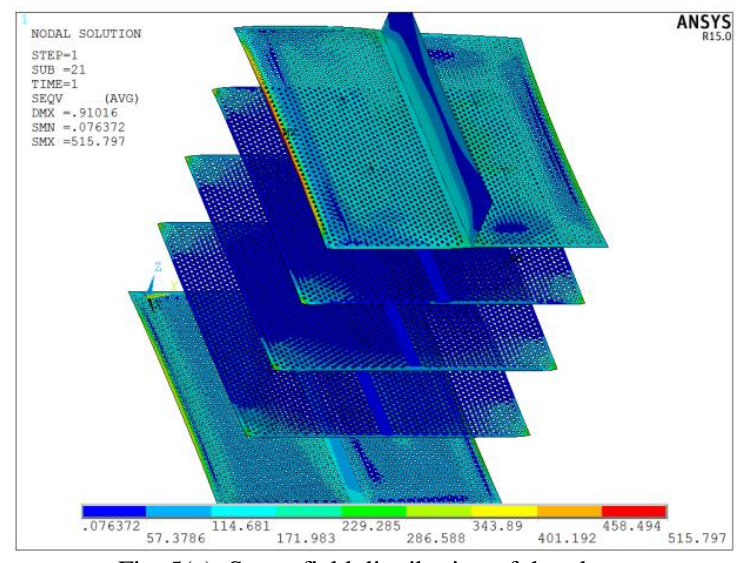

Fig. 5(a). Stress field distribution of the plates.

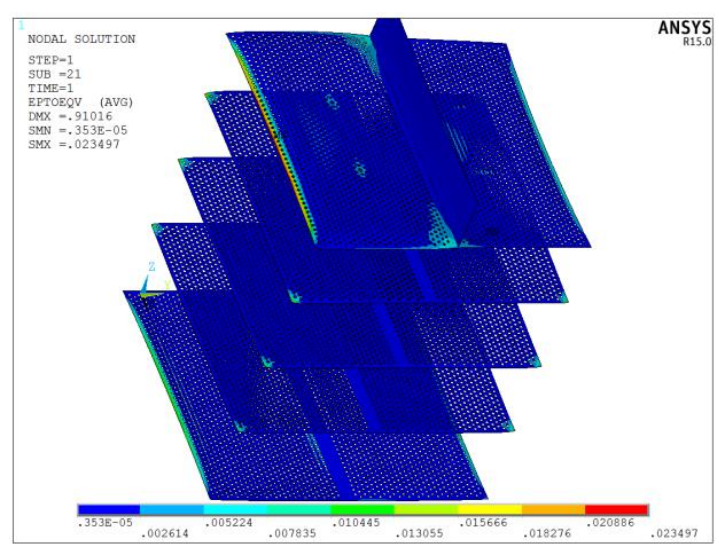

Fig. 5(b). Strain field distribution of the plates.

According to the results of fatigue test, the fatigue curve of the material $1 \mathrm{Cr} 18 \mathrm{Ni} 9 \mathrm{Ti}$ was worked out, the equation of fatigue life of bundle could be sorted as (7):

$$
\Delta \varepsilon_{p} * N_{f}^{0.65515}=1.0351
$$

Based on the result of strain field distribution, the maximum equivalent plastic strain range was obtained, which was plugged in (7), then $N_{f}$ was calculated out. According to the relevant provisions of mechanical design, considering the stress concentration and other factors, setting safety coefficient as 1.5 , the fatigue life calculation result was obtained.

\section{CONCLUSION}

This paper has investigated the failure reason of air heat exchanger and offered a life prediction method of heat exchanger.

Through the result of modal analysis, fluid will induce vibration of tube bundle, which can cause the fatigue damage of the heat exchange tube. At the same time, the modal analysis is also used as the preliminary analysis basis for the next transient dynamics analysis of the tubes.

The result of static structural analysis confirms that thermal stress is one of the major reasons of air heat exchanger fatigue failure. At the tube bundle and the tube sheet contact position, the stress value was obviously higher. Due to the effect of thermal stress and the thermal deformation of tubes, the main failure position of air heat exchanger is tube bundle and the heat exchanger tubes and the plate junction.

The fatigue life of tube bundles under the action of thermal stress was predicted. When the flow field is considered, the stress of each position will increase, the equivalent strain range will increase, and the fatigue life will decrease. Similarly, this model can be used to predict the fatigue life of tube bundles under the simultaneous action of temperature field and flow field.

\section{REFERENCES}

[1] K. A. Gardner, B. Mulgrew, and P. M. Grant, "Heat exchanger tube sheet design," Journal of Applied Mechanics, vol. 4, pp. 377-385, July 1948.

[2] K. P. Singh and M. Hotlz, "An approximate method for evaluating the temperature field in tube sheet laminar of tubular heat exchangers under steady state conditions," Journal of Engineering for Gas Turbines \& Power, vol. 4, pp. 895-900, Jan. 1982.

[3] R. D. Ohol, M. T. Rane, and R. A. Noras, "A finite element procedure for temperature distribution in tube sheets," in Proc. The 7th International Conference of Pressure Vessel Technology, Dusseldorf, 1992, vol. 2, pp. 355-370.

[4] N. Kasahara and K. Iwata, "Simplified 2-dimentional thermal analysis method considering 3-dimensional heat transfer," Computational Mechanism 86, vol. 2, pp. 127-137, 1986.

[5] A. El and S. Amjad, "Hydrogen induced cracking and pitting of brass heat exchanger tube," Materials Science and Technology, vol. 24, pp. 711-717, 2008.

[6] J. X. Wu, Q. W. Dong, and M. S. Liu, "Failure analysis, prevention and on-line inspection of shell and tube heat-exchangers," Pressure Vessel Technology, vol. 18, no. 5, pp. 57-60, June 2001.

[7] C. L. Liu and J. F. Luan, "Analysis of common failure modes of shell and tube heat exchanger and countermeasures discuss," Chemical Equipment Technology, vol. 29, no. 3, pp. 38-40, March 2008.

[8] H. J. Connors, "Fluid elastic vibration of tube arrays excited by cross flow," in Proc. the Symposium on Flow-Induced Vibration in Heat Exchangers, New York, 1970, pp. 42-56.

[9] V. I. Katinas, A. A. Markevicius, V. V. Rodionov, and S. A. Alekseyev, "Transient flow forces induced in heat exchanger tube bundles," Fluid Mechanics-Soviet Research, vol. 18, pp. 44-53, 1989.

[10] Z. C. Wang, B. L. He, and J. H. Han, "Research on multi-field coupling numerical simulation of high temperature heat exchangers," Chemical Engineering \& Machinery, vol. 40, no. 2, pp. 188-192, Feb. 2013.

[11] C. Z. Guo and J. Zhou, "Numerical analysis of the thermal Stress induced by temperature differences of fixed tube-sheet heat exchangers," Chemical Engineering \& Machinery, vol. 36, no. 1, pp. 41-46, Jan. 2009.

[12] M. Z. Xiong, "Calculation of fluid induced vibration in heat exchangers," Journal of Chemical Industry \& Engineering, vol. 28, pp. 150-153, 2007. 
[13] S. W. Qian, W. M. Zeng, S. J. Hong, H. Z. Cen, and M. K. Yuan, "The mechanism of turbulent buffeting in the heat exchanger," Petro-Chemical Equipment, vol. 16, no. 5, pp. 7-11, 1987.

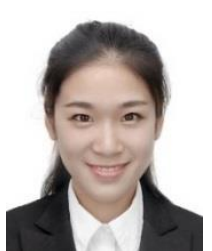

Q. Miao was born in Henan Province, China, in July 1990. Q. Miao graduated from the School of Energy and Power, Dalian University of Technology in Dalian, China, and obtained a bachelor's degree in 2013. At present, Q. Miao is studying for a master's degree in Dalian University of Technology, the main direction is fatigue research.

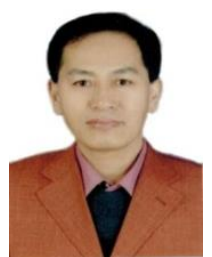

Z. Wang was born in Liaoning Province, China, in July 1960. Z. Wang graduated from Power Engineering Department, Dalian University of Technology in Dalian, China, and obtained a master's degree in 1992 Z. Wang currently serves as a professor of Energy and Power Engineering School, Dalian University of Technology, the main direction is equipment and structural safety evaluation and life prediction.

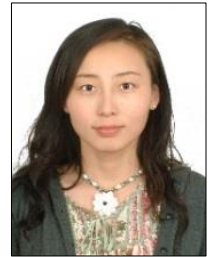

L. Wang was born in Liaoning Province, China, on April 1980. L. Wang graduated from the School of Energy and Power, Dalian University of Technology in Dalian, China, and obtained a doctor's degree in 2012. At present, L. Wang currently serves as a lecturer of Energy and Power Engineering School, Dalian University of Technology, the main direction is equipment and structural safety evaluation and life 